\title{
Endothelial, platelet, and macrophage microparticle levels do not change acutely following transcatheter aortic valve replacement
}

\author{
Julio F. Marchini ${ }^{1,3 *}$, Ayumi Aurea Miyakawa', Flavio Tarasoutchi ${ }^{2}$, José Eduardo Krieger', ${ }^{1}$ Pedro Lemos ${ }^{3}$ \\ and Kevin Croce $^{4}$
}

\begin{abstract}
Background: Patients with severe aortic stenosis have increased levels of prothrombotic and proinflammatory microparticles (MP), and MPs actively regulate pathological processes that lead to atherothrombotic cardiovascular events. Shear stress is a validated stimulus of MP production, and abnormal shear stress in aortic stenosis increases MP release in ex-vivo studies. We hypothesized that in patients with severe aortic stenosis, percutaneous replacement of the aortic valve (TAVR) would reduce abnormal shear stress and would decrease levels of circulating MPs.

Findings: The experimental protocol utilized flow cytometry (FC) and nanoparticle tracking analysis (NTA) to quantify circulating plasma MP levels in aortic stenosis patients at baseline and 5 days after TAVR. The baseline and 5 day MP counts measured by FC were $6.10 \cdot 10^{5} \pm 1.21 \cdot 10^{5} \mathrm{MP} / \mu \mathrm{L}$ and $5.74 \cdot 10^{5} \pm 9.54 \cdot 10^{4} \mathrm{MP} / \mu \mathrm{L}$, respectively $(p=0.91)$. The baseline and 5 day MP counts measured by NTA were $9.29 \cdot 10^{13} \pm 1.66 \cdot 10^{13} \mathrm{MP} / \mathrm{\mu L}$ and $3.95 \cdot 10^{14} \pm 3.11 \cdot 10^{14} \mathrm{MP} / \mu \mathrm{L}$, respectively $(p=0.91)$. When MPs were stratified by cell source, there was no difference in pre/post TAVR endothelial, platelet, or leukocyte MP levels.
\end{abstract}

Conclusion: Levels of circulating MPs do not change acutely following TAVR therapy for aortic stenosis. Trial registered at clinicaltrials.gov NCT02193035 on July 11, 2014.

Keywords: Severe aortic stenosis, Microparticles, Nanoparticle-tracking analysis, Flow cytometry

\section{Findings}

\section{Background}

Microparticles (MPs) are submicron membrane fragments that impair endothelial function, promote thrombosis, and increase the risk of cardiovascular (CV) events [1-5]. Endothelial cells, platelets, and macrophages release MPs in response to inflammatory activation and apoptotic signaling [6-8]. Endothelial and platelet MPs circulate at elevated levels in patients with severe aortic stenosis [9]. Shear stress is a validated stimulus of MP production. Abnormal shear

\footnotetext{
*Correspondence: jfmarchini@usp.br

'Laboratory of Genetics and Molecular Cardiology, Heart Institute, University of São Paulo Medical School, São Paulo, SP, Brazil

${ }^{3}$ Hemodynamics and Interventional Cardiology Service, Heart Institute,

University of São Paulo Medical School, São Paulo, SP, Brazil

Full list of author information is available at the end of the article
}

stress in aortic stenosis increases MP release in ex-vivo studies $[7,9]$. We hypothesized that in patients with severe aortic stenosis, percutaneous replacement of the aortic valve (TAVR) would reduce abnormal shear stress and would decrease levels of circulating plasma MPs.

\section{Methods}

This study included patients with severe aortic stenosis selected for TAVR. Pre-specified exclusions are listed in the Additional file 1. The institutional review board approved the protocol (Comissão de Ética para Análise de Projetos de Pesquisa-CAPPESQ / FMUSP \#12079). The protocol registration number is NCT02193035 (clinicaltrials.gov). All patients provided informed consent to participate in the study. The experimental 
protocol used complementary flow cytometry (FC) and nanoparticle-tracking analysis (NTA) to measure MPs. FC identifies MPs based on size and based on MP affinity for Annexin V. Annexin V binds to the prothrombotic lipid, phosphatidylserine, on the outer layer of the MP surface membrane. FC has a limited ability to detect particles that are smaller than $50-100 \mathrm{~nm}$, and thus FC does not accurately quantify ultra-small MPs [10]. Figure 1 shows representative flow cytometry data of 200nM reference sizing beads (Fig. 1a), Annexin- $\mathrm{V}$ positive MPs (Fig. 1b), EDTA-treated negative controls (Fig. 1b), and cell specific antibodies to subtype and quantify endothelial, platelet, and macrophage MPs (Fig. 1c-e).

NTA identifies all MPs, including those, which do not have phosphatidylserine on their outer surface. Compared to FC, NTA detects MPs with a lower limit of $50 \mathrm{~nm}$ size, and thus NTA has a better sensitivity for ultra-small particles [11]. For this study, MP levels were quantified at baseline and 5 days after TAVR. Detailed methods are available in the Additional file 1.

\section{Results}

We evaluated 15 patients that were enrolled over the course of 12 months and excluded six patients from the study; the reasons for exclusion were fatal stroke (1 patient), fatal cardiogenic shock (1 patient), severe infection (3 patients), and deep venous thrombosis (1 patient). Baseline characteristics of the 9 patients that we analyzed are presented in Table 1.
We identified the cell origin of MPs by detecting antibodies directed against endothelial cells, platelets, and leukocytes. The flow cytometry quantification of MPs pre- and 5-day post-TAVR is shown in Fig. 2. There was no difference in total, endothelial cell, platelet, or macrophage MP counts prior to vs. 5 days after TAVR. Total Annexin $\mathrm{V}$ positive MPs were $6.10 \cdot 10^{5} \pm 1.21 \cdot 10^{5}$ pre-TAVR vs. $5.74 \cdot 10^{5} \pm 9.54 \cdot 10^{4} \mathrm{MP} / \mu \mathrm{L}$ post-TAVR $(p=0.91)$. Endothelial MPs were $2.05 \cdot 10^{5} \pm 3.61 \cdot 10^{4}$ $\mathrm{MP} / \mu \mathrm{L}$ pre-TAVR vs. $2.17 \cdot 10^{5} \pm 3.38 \cdot 10^{4} \mathrm{MP} / \mu \mathrm{L}$ postTAVR $(p=0.73)$. Platelet MPs were $2.79 \cdot 10^{5} \pm 5.02 \cdot 10^{4}$ $\mathrm{MP} / \mu \mathrm{L}$ pre-TAVR vs. $2.74 \cdot 10^{5} \pm 4.41 \cdot 10^{4} \mathrm{MP} / \mu \mathrm{L}$ postTAVR $(p=0.82)$. Macrophage MPs were $2 \cdot 18 \cdot 10^{5} \pm 3.49 \cdot 10^{4}$ $\mathrm{MP} / \mu \mathrm{L}$ pre-TAVR vs. $2.07 \cdot 10^{5} \pm 3.23 \cdot 10^{4} \mathrm{MP} / \mu \mathrm{L}$ postTAVR $(p=0.82)$.

Similar to the FC quantification, there was no difference in NTA-measured MP counts pre vs. 5 days post-TAVR $\left(9.29 \cdot 10^{13} \pm 1.66 \cdot 10^{13} \mathrm{MP} / \mu \mathrm{L}\right.$ pre-TAVR vs. $3.95 \cdot 10^{14} \pm 3.18 \cdot 10^{14} \mathrm{MP} / \mu \mathrm{L}$ post-TAVR, $p=0.91$, Fig. 3a). In our data set, there was no correlation between MP levels measured by FC vs. NTA (Fig. 3b, $\left.r^{2}=0.01\right)$.

Previous investigations have demonstrated that there is a $40 \%$ drop in platelet count following TAVR with a significant incidence of new thrombocytopenia [12]. In our study, the average baseline platelet count was $220 \cdot 10^{9} / \mathrm{L} \pm 90 \cdot 10^{9} / \mathrm{L}$ pre-TAVR, and $183 \cdot 10^{9} / \mathrm{L} \pm 78 \cdot 10^{9} /$ $\mathrm{L}$ post-TAVR $(p=0.0093)$. Platelet counts fell by an average of $17 \%$, and $44 \%$ of patients developed thrombocytopenia.
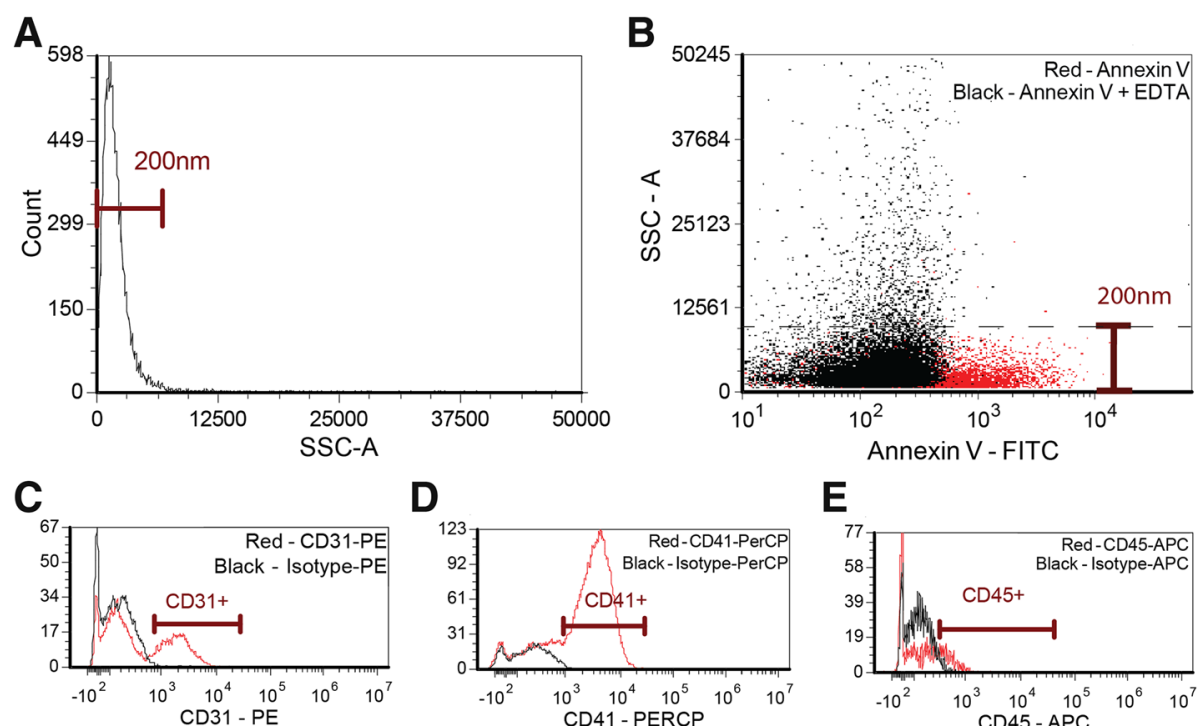

E

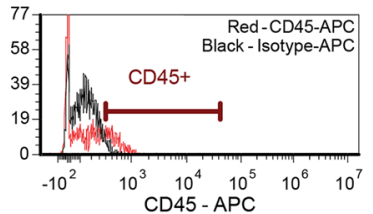

Fig. 1 Microparticle Flow Cytometry Quantification Method. a Side scatter profile of $200 \mathrm{~nm}$ silica size reference beads. b Annexin-V stained microparticles (MP) (red) and Annexin-V stained MPs treated with EDTA (black). The SSC threshold derived from the reference beads is represented by a dashed line (c-e) Representative flow cytometry data of cell specific antibodies to subtype and quantify endothelial, platelet, and macrophage MPs. We defined endothelial MPs as Annexin V+/CD31+ events, platelet MPs as Annexin V+/CD41+ events, and macrophage MPs as Annexin V+/CD45+ events. Red; specific antibody vs. black; isotype control. SSC; side scatter intensity 
Table 1 Patient and procedure characteristics and outcomes

\begin{tabular}{|c|c|}
\hline Characteristics & $N=9$ \\
\hline$\overline{\text { Age }}$ & $84.8 \pm 5.1$ \\
\hline Male & $67 \%$ \\
\hline Euroscore (Logistic) & $23.5 \pm 12.5 \%$ \\
\hline Peripheral artery disease & $11 \%$ \\
\hline End stage renal failure & 0 \\
\hline Creatinine (mg/dL) & $1.52 \pm 0.6$ \\
\hline LV-Ao peak gradient & $66.4 \pm 26.2$ \\
\hline LV-Ao mean gradient & $43.9 \pm 18.2$ \\
\hline $\operatorname{AVA}\left(\mathrm{cm}^{2}\right)$ & $0.7 \pm 0.1$ \\
\hline \multicolumn{2}{|l|}{ Medtronic Corevalve Size } \\
\hline 26 & $22 \%$ \\
\hline 29 & $56 \%$ \\
\hline 31 & $22 \%$ \\
\hline \multicolumn{2}{|l|}{ Outcomes } \\
\hline Post-TAVR LV-AO peak gradient & $13.8 \pm 6.4$ \\
\hline Death within 30 days & 0 \\
\hline Stroke/TIA & 0 \\
\hline $\mathrm{Ml}$ & 0 \\
\hline Pacemaker & $44 \%$ \\
\hline Other Complications & $11 \%$ \\
\hline
\end{tabular}

$L V$ - $A O$ left ventricle-aortic, AVA aortic valve area, TAVR transcatheter aortic valve replacement, $T I A$ transient ischemic attack, $M I$ myocardial infarction, $A F$ atrial fibrillation. Other complications includes arrhythmias, major bleeding and renal failure

\section{Discussion}

Although the sample size could be larger, our initial results predict a futile effort to show a difference in microparticle level pre TAVR vs. 5 days post TAVR. Previous reports demonstrated a $16.8 \%$ decrease in microparticles 90 days post-TAVR [13]. We therefore simulated adding new cases where all the additional cases have a $20 \%$ decrease in microparticle counts. To calculate the likelihood of this scenario we used the distribution of our original sample. In it, we observed a $10 \%$ average increase in flow cytometry (FC) microparticle counts from pre-TAVR to post-TAVR with a standard deviation of $60 \%$. Assuming this is a normal distribution, a $20 \%$ decrease corresponds to half a standard deviation less than the mean. The area of the normal distribution below half a standard deviation is $30.9 \%$, which means that $30.9 \%$ of patients would have at least a $20 \%$ decrease in microparticles. Therefore, the likelihood of a scenario where all six patients present a $20 \%$ decrease in microparticles is $30.9 \%$ to the power of 6 , which is $0.09 \%$.

Next, we did the same modeling for the nanoparticle tracking analysis (NTA). The estimated likelihood of a scenario where all the additional patients would have a decrease of $20 \%$ in microparticle levels is $0.03 \%$. Thus, there is less than $0.1 \%$ chance that increasing the sample size would have an impact on the mean change in microparticle levels for both methods.

Moreover, if we expanded the sample size to 15 patients, and patients number 10 through 15 each had a microparticle level that was $20 \%$ less than their preTAVR level, the $\mathrm{p}$ value for the difference would still be non-significant for both methods (modeled $\mathrm{p}$ value for FC: 0.51 and NTA: 0.13). Therefore, we conclude that there is no justification to expand the study, because doing so would not alter the results or conclusions.

In our investigations, patients with severe aortic stenosis had higher levels of MPs compared to published reports of MP levels in healthy patients. One study that used similar flow cytometry methods found $3.14 \cdot 10^{5} \mathrm{MP} / \mu \mathrm{L}$ (IQR $2.27 \cdot 10^{5}-4.45 \cdot 10^{5} \mathrm{MP} / \mu \mathrm{L}$ ) total MPs [14, 15]. Meanwhile, protocols using NTA methodology report MP levels of $1-5 \cdot 10^{12} \mathrm{MP} / \mu \mathrm{L}$ in healthy controls subjects $[11,16]$. Notably, our finding that FC MP quantification varied by an order of magnitude compared to NTA MP quantification corroborates similar findings from another group [17]. An explanation for the difference in MP counts between the two methods is that unlike FC, NTA quantifies large, small, and ultra-small MPs, as well as both phosphatidylserine positive and negative MPs [17].

Similar to previous reports, we observed marked thrombocytopenia following the TAVR procedure [12]. Platelet consumption has the potential to increase platelet activation and increase associated production of platelet MPs. Despite the potential link between platelet count and MP levels, we saw no relationship between these two parameters in our study (correlation between platelet count, absolute platelet decrease and FC-measured MP, $r^{2}=0.02$ and $r^{2}=0.16$, respectively; correlation between platelet count, absolute platelet decrease and NTAmeasured MP, $r^{2}=0.08$ and $r^{2}=0.06$; NTA, respectively).

This current clinical study demonstrates that circulating MP levels do not change acutely 5 days following TAVR. Although the number of patients enrolled in this study was small, based on the observed means and standard deviation, an increase in sample size would not change the overall conclusions. When interpreting the negative results of this study, it is important to consider the following points: (1) the half-life of circulating vascular MPs is unknown, and if MPs circulate for several days, our brief 5 day follow-up window might prevent detection of changes in MP levels post TAVR, (2) thrombotic or inflammatory processes may be playing a greater role than shear stress in promoting MP elevation in patients with aortic stenosis, (3) the acute stress of the TAVR procedure might promote $\mathrm{MP}$ production-potentially negating the reduction in 

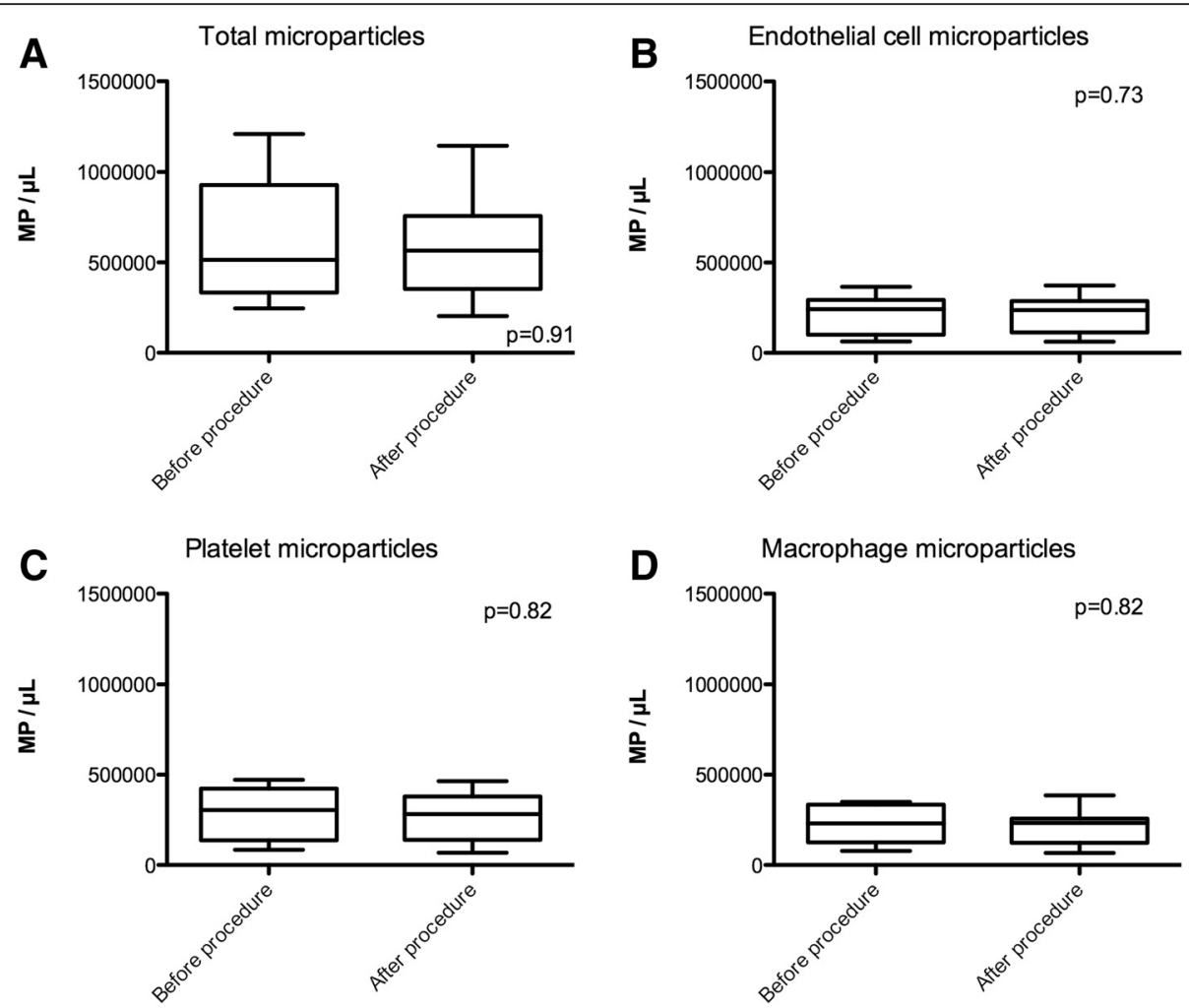

Fig. 2 Flow cytometry measurement of microparticle levels before and 5 days after TAVR procedure. a Total phosphatidylserine positive microparticles, (b) endothelial microparticles, (c) platelet microparticles, and (d) macrophage microparticles. MP; microparticles

MP generation achieved by replacing the stenotic valve, and (4) the 5 day follow-up time that we chose might be too short to enable a resetting of homeostatic systems that activate MP release. Of note, a recent investigation demonstrated that circulating MP levels do decrease 90 days following TAVR [13]. The later time point of 90 days may provide adequate time for recovery from the TAVR procedure and/or resetting of homeostatic systems that promote MP production $[13,18]$.

\section{Conclusions}

Our data found increased MP levels in patients with severe aortic stenosis in comparison to reports of healthy patients. We did not observe a reduction of MP levels in a short follow-up of TAVR. MPs, which have prothrombotic and proinflammatory effects, imply an increased risk of $\mathrm{CV}$ events for patients with severe aortic stenosis that persists acutely after TAVR treatment. Targetting MP generation or effects could reduce TAVR periprocedural CV events.

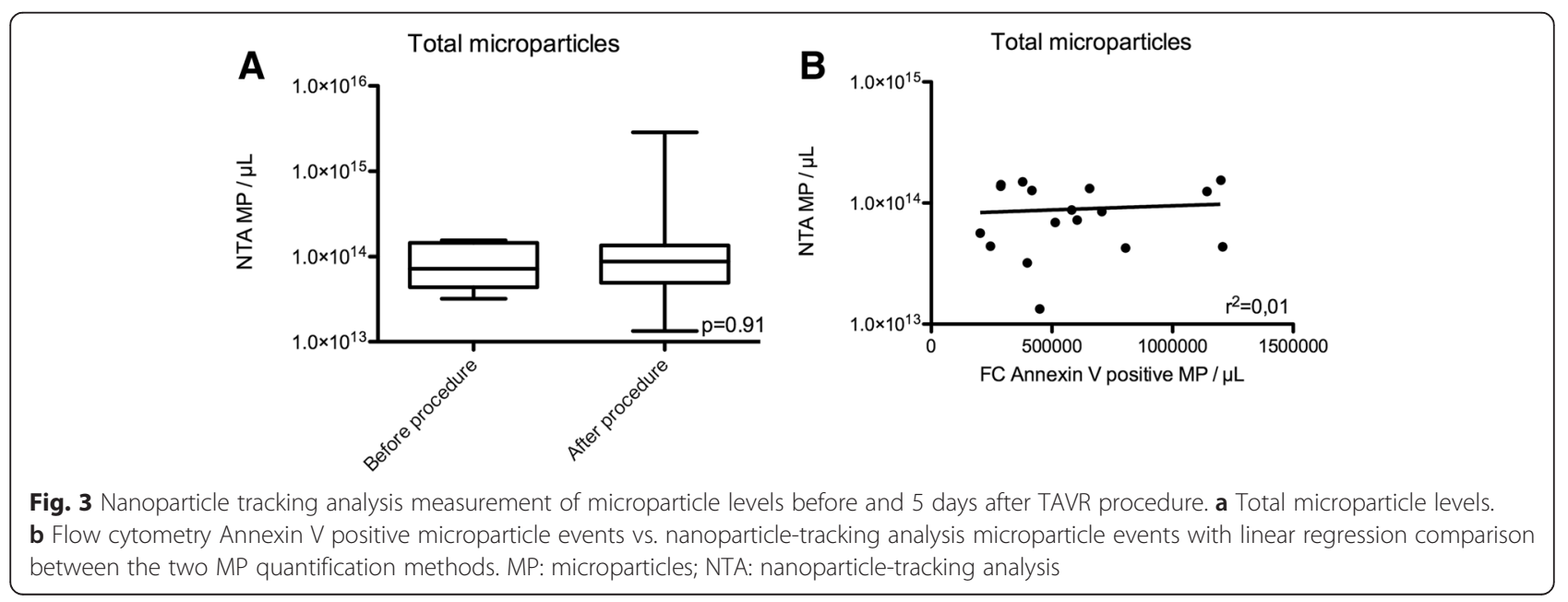




\section{Additional file}

Additional file 1: Detailed description of the methods. (DOCX $25 \mathrm{~kb}$ )

\section{Abbreviations}

FC: flow cytometry; MP: microparticle; TAVR: transcatheter aortic valve replacement; NTA: nanoparticle-tracking analysis.

\section{Competing interests}

The authors declare no competing interest.

\section{Authors' contributions}

JM, AAM, FT, JEK, PL and KC contributed to the design of the study and its protocol. PL performed the TAVR procedures. JM acquired the samples, performed the experiments. JM and KC wrote the article. KC revised the article. JM, AAM, FT, JEK, PL and KC read the manuscript and approved the final version.

\section{Acknowledgments}

Fundação de Amparo à Pesquisa do Estado de São Paulo (BR) provided funding for this project to J.E.K \#2013/17368-0.

\section{Author details}

${ }^{1}$ Laboratory of Genetics and Molecular Cardiology, Heart Institute, University of São Paulo Medical School, São Paulo, SP, Brazil. Valvular Heart Disease Unit, University of São Paulo Medical School, São Paulo, Brazil.

${ }^{3}$ Hemodynamics and Interventional Cardiology Service, Heart Institute, University of São Paulo Medical School, São Paulo, SP, Brazil. ${ }^{4}$ Cardiovascular Division, Department of Medicine, Brigham and Women's Hospital, Harvard Medical School, Boston, MA, USA.

\section{Received: 24 October 2015 Accepted: 1 April 2016}

\section{Published online: 11 April 2016}

\section{References}

1. Boulanger CM, Scoazec A, Ebrahimian T, Henry P, Mathieu E, Tedgui A Mallat Z. Circulating microparticles from patients with myocardial infarction cause endothelial dysfunction. Circulation. 2001;104:2649-52.

2. Pfister SL. Role of platelet microparticles in the production of thromboxane by rabbit pulmonary artery. Hypertension. 2004;43:428-33.

3. Toti F, Satta N, Fressinaud E, Meyer D, Freyssinet JM. Scott syndrome, characterized by impaired transmembrane migration of procoagulant phosphatidylserine and hemorrhagic complications, is an inherited disorder. Blood. 1996;87:1409-15.

4. Huber J, Vales A, Mitulovic G, Blumer M, Schmid R, Witztum JL, Binder BR, Leitinger N. Oxidized membrane vesicles and blebs from apoptotic cells contain biologically active oxidized phospholipids that induce monocyte-endothelial interactions. Arterioscler Thromb Vasc Biol. 2002;22:101-7.

5. Viera AJ, Mooberry M, Key NS. Microparticles in cardiovascular disease pathophysiology and outcomes. J Am Soc Hypertens. 2012;6:243-52.

6. Jimenez JJ, Jy W, Mauro LM, Horstman LL, Soderland C, Ahn YS. Endothelial microparticles released in thrombotic thrombocytopenic purpura express von Willebrand factor and markers of endothelial activation. Br J Haematol. 2003;123:896-902.

7. Nomura S, Tandon NN, Nakamura T, Cone J, Fukuhara S, Kambayashi J. High-shear-stress-induced activation of platelets and microparticles enhances expression of cell adhesion molecules in THP-1 and endothelial cells. Atherosclerosis. 2001;158:277-87.

8. Mesri M, Altieri DC. Leukocyte microparticles stimulate endothelial cell cytokine release and tissue factor induction in a JNK1 signaling pathway. J Biol Chem. 1999:274:23111-8.

9. Diehl P, Nagy F, Sossong V, Helbing T, Beyersdorf F, Olschewski M, Bode C, Moser M. Increased levels of circulating microparticles in patients with severe aortic valve stenosis. Thromb Haemost. 2008:99:711-9.

10. van der Pol E, Hoekstra AG, Sturk A, Otto C, van Leeuwen TG, Nieuwland R. Optical and non-optical methods for detection and characterization of microparticles and exosomes. J Thromb Haemost. 2010;8:2596-607.

11. Dragovic RA, Gardiner C, Brooks AS, Tannetta DS, Ferguson DJ, Hole P, Carr B, Redman CW, Harris AL, Dobson PJ, et al. Sizing and phenotyping of cellular vesicles using Nanoparticle Tracking Analysis Nanomedicine. 2011;7:780-8.

12. McCabe JM, Huang PH, Riedl LA, Devireddy SR, Grondell J, Connors AC, Davidson MJ, Eisenhauer AC, Welt FG. Incidence and implications of idiopathic thrombocytopenia following transcatheter aortic valve replacement with the Edwards Sapien ((c)) valves: a single center experience. Catheter Cardiovasc Interv. 2014;83:633-41.

13. Horn P, Stern D, Veulemans V, Heiss C, Zeus T, Merx MW, Kelm M, Westenfeld R. Improved endothelial function and decreased levels of endothelium-derived microparticles after transcatheter aortic valve implantation. Eurolntervention. 2015;10:1456-63.

14. Jy W, Horstman LL, Jimenez JJ, Ahn YS, Biro E, Nieuwland R, Sturk A, Dignat-George F, Sabatier F, Camoin-Jau L, et al. Measuring circulating cell-derived microparticles. J Thromb Haemost. 2004;2:1842-51.

15. Hron G, Kollars M, Weber H, Sagaster V, Quehenberger P, Eichinger S, Kyrle PA, Weltermann A. Tissue factor-positive microparticles: cellular origin and association with coagulation activation in patients with colorectal cancer. Thromb Haemost. 2007;97:119-23.

16. Gardiner C, Ferreira YJ, Dragovic RA, Redman CW, Sargent IL: Extracellular vesicle sizing and enumeration by nanoparticle tracking analysis. J Extracell Vesicles. 2013;2:19671.

17. van der Pol E, Coumans FA, Grootemaat AE, Gardiner C, Sargent IL, Harrison P, Sturk A, van Leeuwen TG, Nieuwland R. Particle size distribution of exosomes and microvesicles determined by transmission electron microscopy, flow cytometry, nanoparticle tracking analysis, and resistive pulse sensing. J Thromb Haemost. 2014;12:1182-92.

18. Petrov G, Regitz-Zagrosek V, Lehmkuhl E, Krabatsch T, Dunkel A, Dandel M, Dworatzek E, Mahmoodzadeh S, Schubert C, Becher E, et al. Regression of myocardial hypertrophy after aortic valve replacement: faster in women? Circulation. 2010;122:S23-28.

\section{Submit your next manuscript to BioMed Central} and we will help you at every step:

- We accept pre-submission inquiries

- Our selector tool helps you to find the most relevant journal

- We provide round the clock customer support

- Convenient online submission

- Thorough peer review

- Inclusion in PubMed and all major indexing services

- Maximum visibility for your research

Submit your manuscript at www.biomedcentral.com/submit 